\title{
Study on Imaging Quality of Water Ball Lens
}

\author{
Haiyan Yang ${ }^{1, \mathrm{a},{ }^{*}}$, Xiaopan $\mathrm{Li}^{1, \mathrm{~b}},{ }^{1, \mathrm{c}}$ Hao Kong , 1,d Guangyang Xu and ${ }^{1, \mathrm{e}}$ Yan Cai \\ ${ }^{1}$ School of Physics and Information Engineering, Zhaotong University, Zhaotong 657000, China; \\ a elaincoco@foxmail.com, bztu_lxp@foxmail.com, \\ cynkonghao@gmail.com, ${ }^{\mathrm{d}} 10344843 @ q q . c o m,{ }^{\mathrm{e}}$ china_ycai@163.com
}

Keywords: water ball lens, geometrical aberration, diffraction effects, large field of view

\begin{abstract}
The water ball lens is a simple, thick convex lens made of glass balls filled with high purity water. In this paper, the imaging quality of water ball lens is quantitatively analyzed by ZEMAX optical design software. The analysis results show that the water ball lens has the characteristics of large field of view and good imaging quality, so it can meet the needs of astronomical observation in large field of view.
\end{abstract}

\section{Introduction}

ZEMAX is an optical design software developed by Radiant ZEMAX company of America. This is a set of optimization, tolerance and other analysis functions software. It can complete the design of optical components, simulate sequential and Non-Sequential, and analyze illumination of lighting system $^{[1,3]}$. ZEMAX can also build optical models such as reflection, refraction and diffraction.

This paper applies ZEMAX software to simulate water ball lens imaging, let us have a more intuitive understanding of the ball lens imaging, the effective focal length calculation of paraxial optical has become more simple, like the spot size can be read directly by optical analysis software. Therefore, ZEMAX software has become an important tool for imaging of water ball lens.

\section{Resolution of Water Ball Lens}

The water ball lens is a simple, thick convex lens made of glass balls filled with high purity water. We all know that convex lenses have a converging effect on light, and that the larger the size of a convex lens used for $\gamma$ astronomical observations, the better. But convex lenses of large enough size in real life are rare and expensive. The combination of glass spheres and high pure water can simply help us achieve the focusing effect of convex lenses. The water ball lens has the characteristics of large field of view and good imaging quality.

The resolving power of lens is the minimum distance between two points of an object that can be distinguished from an imaged object. Resolution is the closest distance between two points, still the minimum distance between two points. The main factors affecting the resolution of water crystal lenses are aberrations and diffraction effects.

\subsection{The Effect of Aberration on Resolution}

Aberration is the deviation of the position and shape of an image from an optical instrument with that of an ideal image. The aberration is mainly divided into geometrical aberration and chromatic aberration. The water ball lens has good spherical symmetry. In this paper, the influence of astigmatism on the resolution is neglected, mainly considering the influence of spherical aberration and chromatic aberration.

Spherical aberration, spherical aberration is due to the intersection, through the lens of different refractive light band and misalignment caused by shaft, which originally is a geometric object point by point focusing optical system, and optical axis angle of light to different optical axis at different positions, thus forming a defocusing circular spot radius is $\mathrm{R}_{\mathrm{A}}{ }^{[4]}$, as shown in Figure 1 . For a water ball lens, the nearer the lens's rays to its edge band, the closer it is to the lens after refraction. 
Usually, the distance between the closest (or closest) intersection of the light and the axis and the intersection point between the paraxial ray and the principal axis is used to represent the magnitude of the spherical aberration. The radius of curvature of the lens surface and the refractive ${ }^{[5]}$ index of the lens material are the important factors that affect the spherical aberration of the water ball lens.

If only considering the aberrations, as shown in Fig 1 , the off-axis light in the distance near focus lens optical center, as shown in the I plane. Paraxial light in the distance far focus lens optical center, as shown in the II plane. The far axis light of a lens has a focal difference when it is imaged with paraxial light. As the plane moves between the far axis image plane and the paraxial image plane, there is a minimum focal spot, with a radius of RA. When the distance between the far axis image plane and the paraxial image plane decreases, the RA becomes smaller and the spherical aberration becomes smaller.

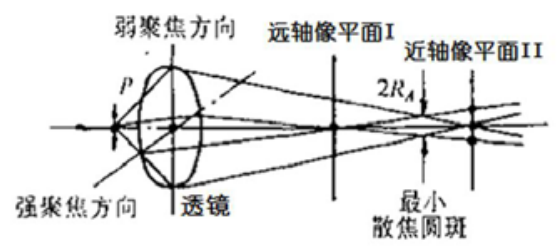

Fig 1 Sketch of far axis focusing and paraxial focusing of lens.

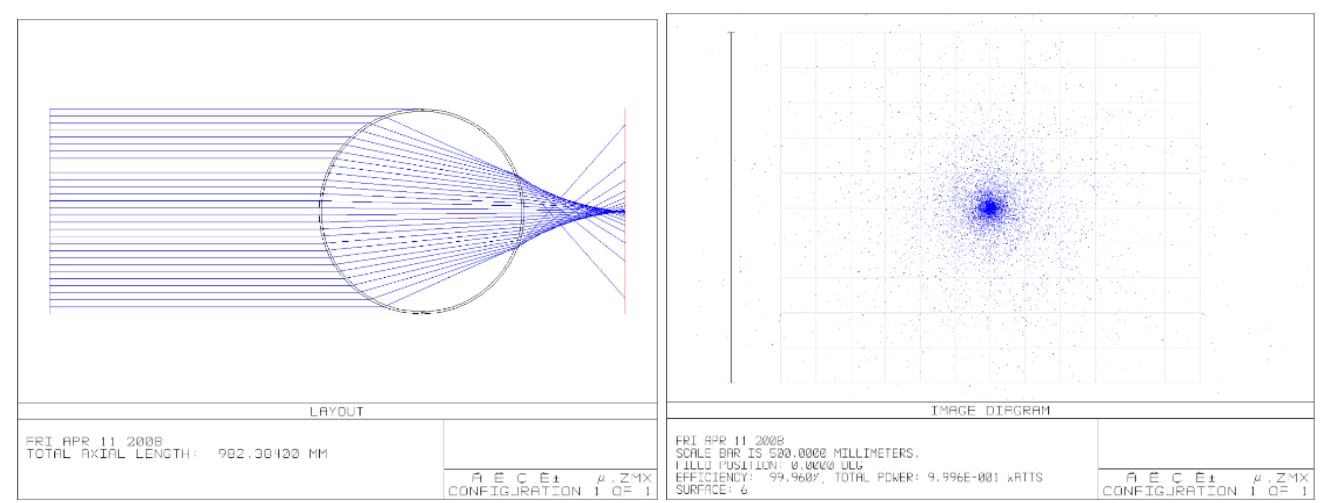

Fig 2 (on left) Water ball lens imaging light path.

Fig 3 (on right) Distribution of light on focal plane with incident light.

ZEMAX defines the distance of the effective focal length from the posterior principal axis to the paraxial image surface, which is the conjugate distance of the infinite object. The calculation of the main surface is usually based on the data of near axis light entering the interface of different refractive indexes ${ }^{[6]}$.In this paper, the refractive index of air is 1 . The use of water ball lens imaging optical path diagram of the ZEMAX software to draw as shown in Fig 2, spherical shell in BK7 glass, the refractive index is 1.52, the ball is filled with refractive index of 1.33 water (due to wavelength in the range of $350 \sim 1100 \mathrm{~nm}$, the water refractive index is about 1.3 , thus ignoring the efficiency of water with the change of wavelength ${ }^{[7]}$ ), collimated monochromatic light incident on the image plane, effective focal plane (i.e. a far distance center effective focal plane for non-paraxial system may not be accurate enough), water ball center distance lens $347.4 \mathrm{~mm}$. After the refraction of the water ball lens, the light far away from the optical axis is caused by the spherical aberration of the water ball lens. It can be seen, not in the collimated monochromatic light incident when all light can be focused to a point, but the formation of a relatively dispersed spot in the effective focal plane, as shown in Fig3, like the size of the plane is $500 \mathrm{~mm} * 500 \mathrm{~mm}$. In addition to the spherical aberration, water ball lens is the same as the ordinary lens, with different wavelength light through the lens due to different refractive index, the image will have position deviation, such as color light (white light irradiation) hollow "cross" (as shown in Fig 4) and the image with color the edge is the phenomenon of color, as shown in Fig 5. 


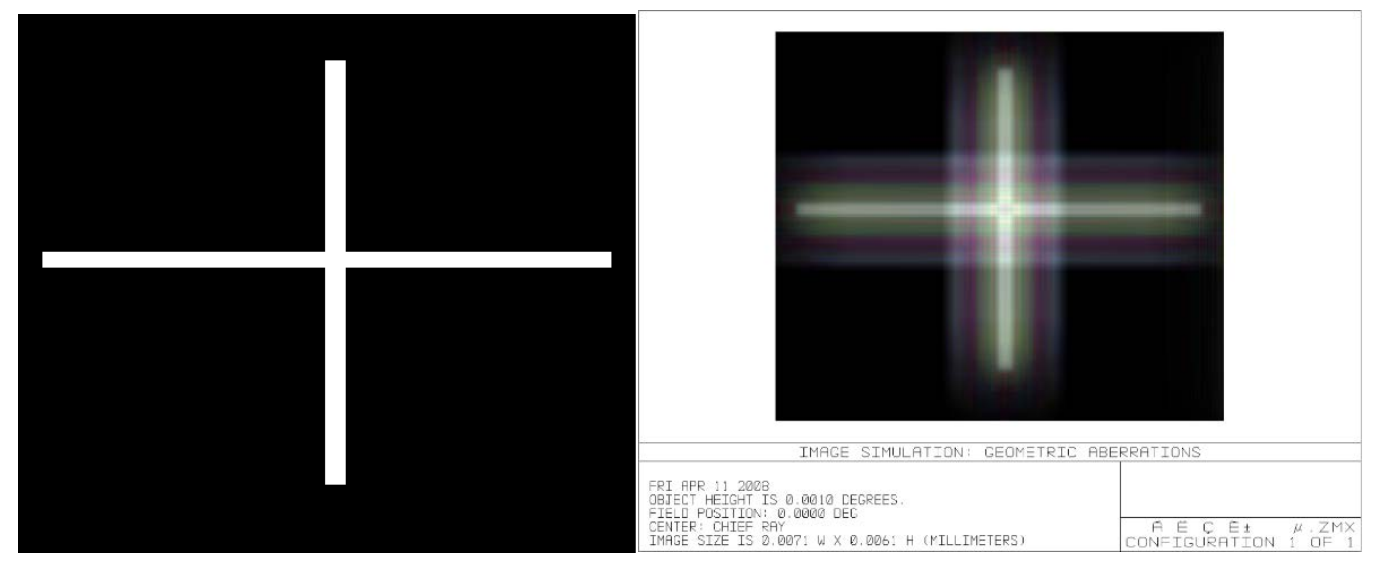

Fig 4 (on left) The front of the water ball lens serves as the "cross" for the light source. Fig 5 (on right) The image of the compound "cross" light source through the water ball lens.

\subsection{Diffraction Effect on Resolution}

In 1870, diffraction effects were used by Abbe to indicate the resolving limit of optical lenses. The resolution of a lens is to distinguish two points close to each other, still imaging the minimum distance from two points. If this distance is set to $\mathrm{D}$, then there is the Abbe formula

$$
d=\frac{0.61 \lambda}{n \sin \theta}
$$

In the formula, $\lambda$ is the wavelength of incident light, $\mathrm{n}$ is the relative refractive index of the medium, $\theta$ is the lens aperture angle. By formula (1), the smaller the $\mathrm{D}$, the higher the resolution of the lens. In the medium and the light source remains unchanged, without considering the influence of aberration on the resolution, while opening half angle $\theta$ is larger, the higher the resolution of lens.

To sum up, and affected by the resolution of a lens aberration and diffraction effect, and the aperture angle of $\theta$ effect on two cases of resolution is the opposite: Reduce the spherical aberration, can reduce the aperture width of $\theta$, but $\theta$ decreases $\mathrm{D}$, and decrease the resolution of lens. When we need to pursue the perfect resolution, must be both, the key is to determine the optimal aperture width of $\theta$, when the diffraction effect of Airy spot and spot size equal to the size of the defocus aberration, their effect on lens effect. Semi angle aperture $\theta$ is larger, then the field of view is more big, if you want the best resolution, you need to lose a certain field to achieve.

\section{Conclusions}

Water ball lens imaging simulation of $35 \mathrm{~cm}$ diameter by ZEMAX, water ball lens is made of glass (BK7) shell $(0.4 \mathrm{~cm}$ thickness, refractive index 1.52) and water (the refractive index is about 1.33). The effective focal length lens, lens to get water ball under the near axis condition is about $34.7 \mathrm{~cm}$ (from the center). If the off-axis light is incident, the focal length will be less than the effective focal length.

The monochromatic "cross" light source is used. The simulation results show that the incident of monochromatic light has the influence of spherical aberration (there is halo in the cross center), and there is no chromatic aberration, and the outline is clearly visible, as shown in Fig 6. 


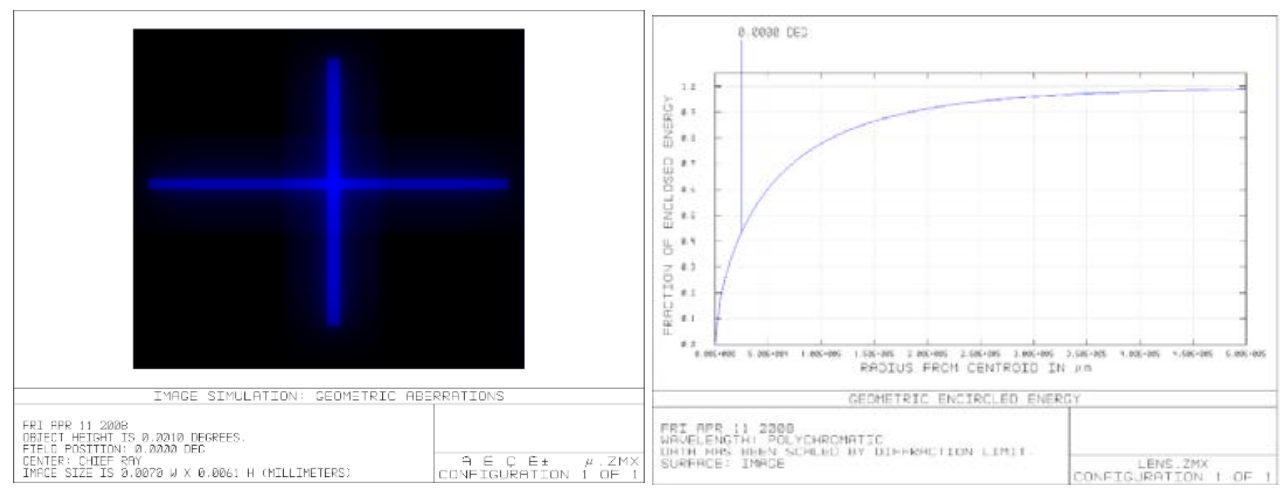

Fig 6 (on left) The image of a monochromatic "cross" light source.

Fig 7 (on right) Circle radius: energy curve.

The total energy is nearly 1 axis monochromatic parallel light, in the focal plane of the image formed by circular spots, including different energy percentage of concentric circles of different radii, called the concentric circle is surrounded by the corresponding energy, the effective focal plane of the enclosing circle: The energy curve is shown in Fig 7

For water ball lenses, the size of the spot is one of our most important concerns, and its size directly affects the size of the photodetector. When the total energy of the incident monochromatic parallel light is 1 , the section diameter of the light in the square surface of the water ball lens is defined as pupil diameter, and is represented by $\mathrm{D}$. The enclosing circle enclosing the total energy $68 \%$ on the effective focal plane is called the image spot, and the size of the spot is described by the radius of the surrounding circle. The size of the detector is determined by the size of the spot.

Simulation of $35 \mathrm{~cm}$ diameter water ball lens, the simulation results, such as spot size with pupil diameter $\mathrm{D}$ changes in Table 1 shows that the curve shown in Fig 8 shows that the spot size increases with pupil diameter. From the above simulation results, the ideal imaging effect of water ball lens, suitable for precision astronomical observation is slightly lower, can satisfy the requirement of large field of view of astronomical observations, and can achieve the requirements of astronomical observation precision, is an ideal equipment for observation at the present stage.

Table 1 The relationship between image spot size and pupil diameter

\begin{tabular}{|c|c|c|c|c|c|c|c|}
\hline $\mathrm{D} / \mathrm{cm}$ & 35 & 30 & 25 & 20 & 15 & 10 & 5 \\
\hline spot size/cm & 6.7 & 3.3 & 1.7 & 0.8 & 0.3 & 0.1 & 0.02 \\
\hline
\end{tabular}

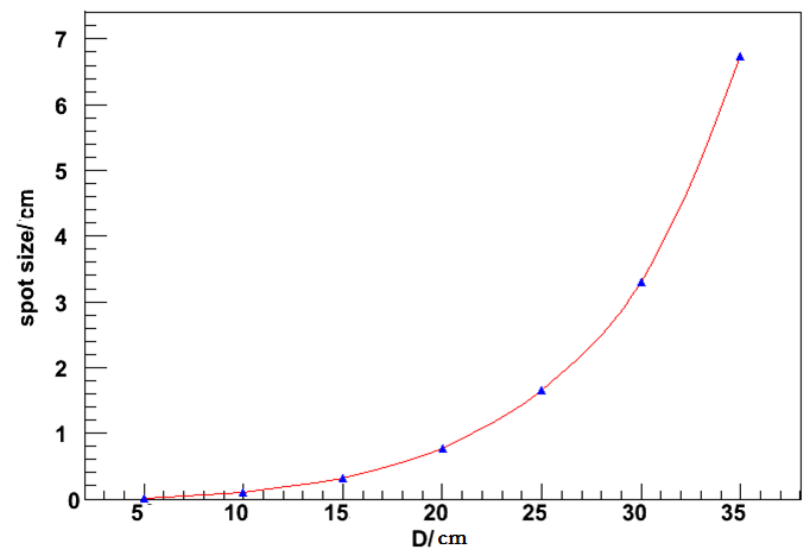

Fig 1 The change curve of image radius spot, size with pupil diameter D

\section{References}

[1] Information on http://baike.baidu.com/view/996468.htm 
[2] Y.H. Ding, Experimental study of hyperspectral imaging system based on acousto-optic tunable filter. Harbin: Harbin Institute of Technology,(2011)19-20

[3] Information on http://zh.wikipedia.org/wiki/Zemax.

[4] Q.J.Yao, Optics course, higher education press, Beijing, 2006,pp.273-277.

[5] Z.D. Jiang, The aberration of lenses is discussed by using EXCEL software. Journal of Dalian Institute of Education.01 (2006), 26-27.

[6] Information on http://www.docin.com/p-15299600.html

[7] G.L. Jiang, P. Sun, A model for the relationship between refractive index and wavelength of water. Chinese Journal of Spectroscopy Laboratory.04(2002), 554-556. 